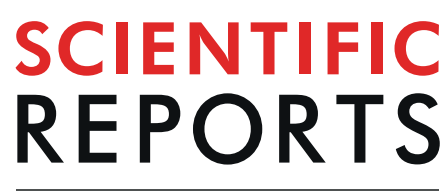

natureresearch

\title{
OPEN Lithium-ion Battery Thermal Safety by Early Internal Detection, Prediction and Prevention
}

Received: 5 June 2019

Accepted: 28 August 2019

Published online: 13 September 2019
Bing Li ${ }^{1}$, Mihit H. Parekh², Ryan A. Adams ${ }^{2}$, Thomas E. Adams ${ }^{3}$, Corey T. Love ${ }^{4}$, Vilas G. Pol ${ }^{2}$ \& Vikas Tomar $^{1}$

Temperature rise in Lithium-ion batteries (LIBs) due to solid electrolyte interfaces breakdown, uncontrollable exothermic reactions in electrodes and Joule heating can result in the catastrophic failures such as thermal runaway, which is calling for reliable real-time electrode temperature monitoring. Here, we present a customized LIB setup developed for early detection of electrode temperature rise during simulated thermal runaway tests incorporating a modern additive manufacturing-supported resistance temperature detector (RTD). An advanced RTD is embedded in a 3D printed polymeric substrate and placed behind the electrode current collector of CR2032 coin cells that can sustain harsh electrochemical operational environments (acidic electrolyte without Redox, short-circuiting, leakage etc.) without participating in electrochemical reactions. The internal RTD measured an average $5.8^{\circ} \mathrm{C}$ higher temperature inside the cells than the external RTD with almost 10 times faster detection ability, prohibiting thermal runaway events without interfering in the LIBs' operation. A temperature prediction model is developed to forecast battery surface temperature rise stemming from measured internal and external RTD temperature signatures.

Lithium-ion batteries (LIBs) have a profound impact on the modern industry and they are applied extensively in aircraft, electric vehicles, portable electronic devices, robotics, etc. ${ }^{1-3}$. However, LIBs are prone to failure due to overheating, over-discharging, overcharging or short-circuit ${ }^{4}$. During such abusive events, chemical energy quickly converts to thermal energy, which leads to heat accumulation, then thermal runaway, fire and explosion ${ }^{5}$. Although there are various safety installations at present such as pressure burst discs, shutdown separators, and one-shot fuses, severe battery hazards have been reported repeatedly ${ }^{4}$. This calls for an in-service thermal monitoring approach. One simple way applied intensively is to measure battery surface temperature ${ }^{6,7}$. Despite the simplicity, external surface temperature monitoring has been shown ineffective due to dissipated heat for LIB safety management ${ }^{8}$. Temperature difference within LIB during battery failure impairs reliability and efficiency of surface temperature based safety management. Finegan et al. analyzed short-circuit condition of 18650 LIB cells with infrared camera and the temperature rise occurred asynchronously across the cell ${ }^{9}$. Liu et al. and Zhao et al. simulated temperature evolution during short circuit of LIB with finite element method, and they observed significant temperature gradient in both thickness and height direction of shorted Li-ion pouch cells ${ }^{10,11}$. A comparison of electrode and battery surface temperature showed that the external surface-based measurement detected peak temperature with reduced magnitude and time delay, and electrode temperature should be the key for prevention of thermal runaway ${ }^{8}$. Zhang et al. introduced internal short-circuit to LIB with memory alloy, and it took $18 \mathrm{~s}$ on average for battery surface temperature to reach peak value after short circuit was triggered ${ }^{12}$. For real-time electrode temperature monitoring, Zhang et al. used a T-type thermocouple inserted between the anode and separator of $18650 \mathrm{LIB}^{8}$. Electrode temperature during battery short-circuit was recorded and compared with battery surface temperature. Novais et al. inserted a fiber bragg grating sensor between double layer separators of the pouch cell to measure temperature change in-operando ${ }^{13}$. During cycling, fluctuation in the electrode temperature was reported.

Such internal sensor-based electrode temperature measurements have offered superior temperature measurement efficiency and accuracy. It has also been applied with widely adopted short circuit tests for LIB safety

${ }^{1}$ School of Aeronautics and Astronautics, Purdue University, West Lafayette, IN, 47907, USA. ${ }^{2}$ Davidson School of Chemical Engineering, Purdue University, West Lafayette, IN, 47907, USA. ${ }^{3}$ Naval Surface Warfare Center, Crane Division, Crane, IN, 47522, USA. ${ }^{4}$ U.S. Naval Research Laboratory, Washington, DC, 20375, USA. Correspondence and requests for materials should be addressed to V.G.P. (email: vpol@purdue.edu) orV.T. (email: tomar@purdue.edu) 
(a)

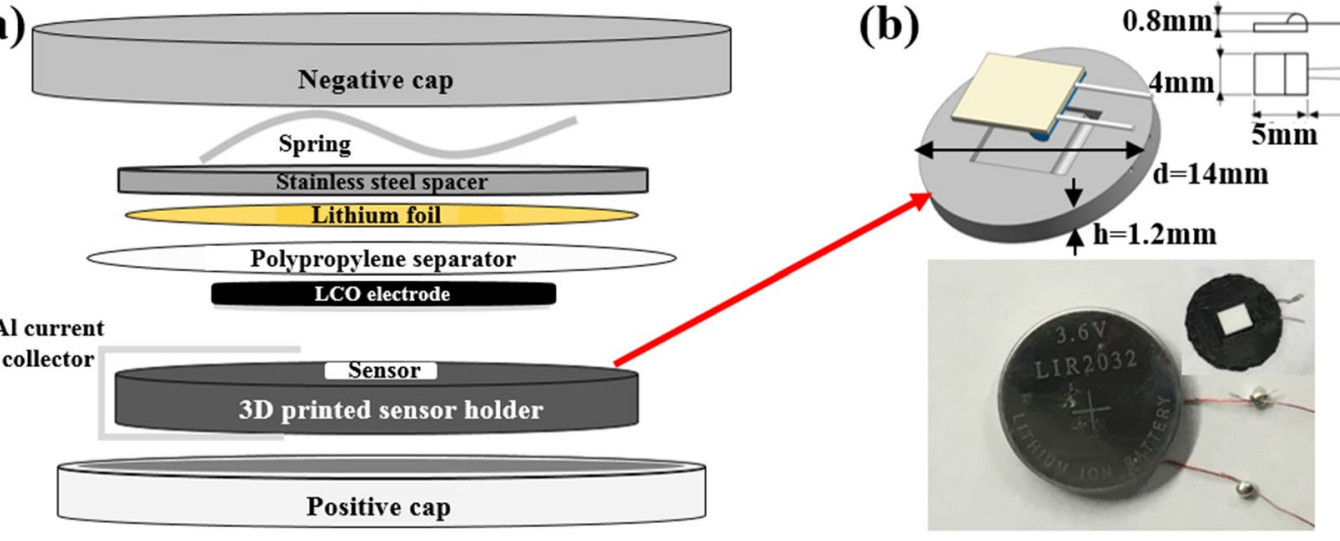

Figure 1. (a) Schematic of customized RTD embedded LIB coin cell; (b) RTD embedded PLA spacer and CR2032 cell with internal RTD. Dimension of the RTD embedded spacer was comparable to ordinary CR2032 coin cell spacer, allowing for reliable sensor-electrode contact and cell sealing after assembly.

analysis, where the shorted battery are subjected to risk of thermal runaway, fire and explosion ${ }^{14,15}$. When the examined LIBs were shorted to simulate thermal runaway conditions, temperature differences up to $50^{\circ} \mathrm{C}$ were observed between the internal and external thermocouples, and the internal thermocouple reported the peak temperature nearly $20 \mathrm{~s}$ in advance ${ }^{8}$. However, it was noted that the sensor embedded between the cathode and anode ${ }^{8,13}$ may impede electrochemical reaction during the battery operation. It is challenging to maintain the contact between porous electrode material and a sensor without damaging the electrode, as microcracks are prone to form in porous structure under compression and lead to material fracture ${ }^{16}$. During battery thermal hazards such as a thermal runaway phenomena, violent temperature rise leads to cracking of the electrode material ${ }^{17}$ and other particle based structures ${ }^{18}$, which can impair the contact between the sensor and electrode material. Our previous work also showed that direct mechanical load influences the electrochemical performance of $\mathrm{LiCoO}_{2}$ (LCO) cathode significantly ${ }^{19}$, implying that inserting a sensor between the electrodes may not be preferable.

In this work, a novel method for incorporating a resistance temperature detector (RTD) behind the cathode current collector of a LIB via additive manufacturing was developed for electrode damage minimization and internal LIB in-operando temperature measurement efficiency improvement. Customized LIBs (CR2032 coin cells) were tested for structural and electrochemical stability in vibrational loading environments. The thermal hazard detecting capability was evaluated using intentional heat rise due to an applied external short circuit ${ }^{8}$. Internal RTD placement yielded significantly superior measuring efficiency and accuracy in comparison to literature reports.

\section{Results}

RTD sensor embedded lithium-ion coin cell for electrode temperature measurement. For the CR2032 coin cells employed in this work, the RTD was incorporated into a customized polylactic acid (PLA) spacer with additive manufacturing, which was placed beneath the cathode as shown in Fig. 1. Sensor placement on the anode side is being addressed in a separate work. Temperature measuring efficiency and accuracy as well as electrochemical stability of customized spacer were analyzed and details of these analyses are provided in the Method section.

The result of hot stage temperature measurement with RTD embedded spacer is presented in Fig. 2a, where the RTD embedded spacers were clamped onto the hot stage at $t=20 \mathrm{~s}$. As shown, the customized spacer provides temperature readings with an error $<1{ }^{\circ} \mathrm{C}$ up to $55^{\circ} \mathrm{C}$, and an average error of $0.82^{\circ} \mathrm{C}$. The response rate of the spacer is evaluated in Figs. $2 b, c$, where the response time $t_{90}$ is defined as the time required for RTD to capture $90 \%$ of the total temperature shift ${ }^{20}$. The average value of $\mathrm{t}_{90}$ is $5 \mathrm{~s}$ for the RTD embedded spacer, which agrees with the observation for the RTD response rate. The $t_{90}$ also presents no dependency on target temperature within the assessed temperature range. These results indicate that the RTD embedded spacer could detect thermal hazards with high efficiency and has limited measuring error over the temperature range covering room temperature to the onset temperature of LIB thermal runaway ${ }^{21}$.

In Fourier-transform infrared (FTIR) spectral analysis of RTD embedded spacer inertness, there are no changes in band intensity and frequency of the electrolyte as shown in Fig. 2d. The consistent FTIR spectra indicate that there is no transition in electrolyte composition and concentration ${ }^{22}$, and the RTD embedded spacer is electrochemically inert, avoiding interference to LIB operation. During cycling of the RTD embedded cell, based on the NAVSEA 9310 vibration test specifications, the LIB cell presents consistent charge/discharge behavior and the cell structure is robust under applied vibrational load.

Customized CR2032 coin cells with $12.5 \mathrm{~mm}$ diameter electrodes were prepared as discussed previously. Cells were cycled between $3.0 \mathrm{~V}$ and $4.3 \mathrm{~V}$ (Fig. $2 \mathrm{e}$ ) using "C/12 rate". Cells report a discharge capacity of about 120 $\mathrm{mAh} \mathrm{g}^{-1}$ and a charge capacity of about $140 \mathrm{mAh} \mathrm{g}^{-1}$ as shown in Fig. 2e. First cycle capacity of the cell is slightly lower due to the formation of a passivation layer on the surface of the cathode ${ }^{23}$. Following that, the charge and discharge profiles overlapped well for the next cycles. During charging, delithiation starts at around 3.9 V; while 
(a)

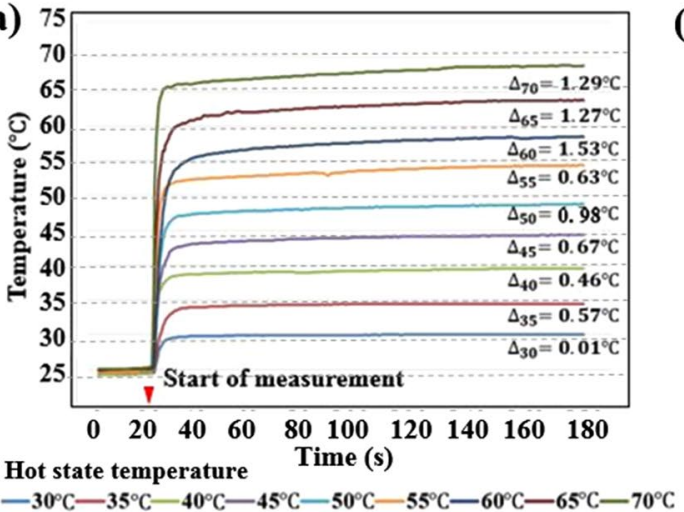

$-30^{\circ} \mathrm{C}-35^{\circ} \mathrm{C}-40^{\circ} \mathrm{C}-45^{\circ} \mathrm{C}-50^{\circ} \mathrm{C}-55^{\circ} \mathrm{C}-60^{\circ} \mathrm{C}-65^{\circ} \mathrm{C}-70^{\circ} \mathrm{C}$

(c)

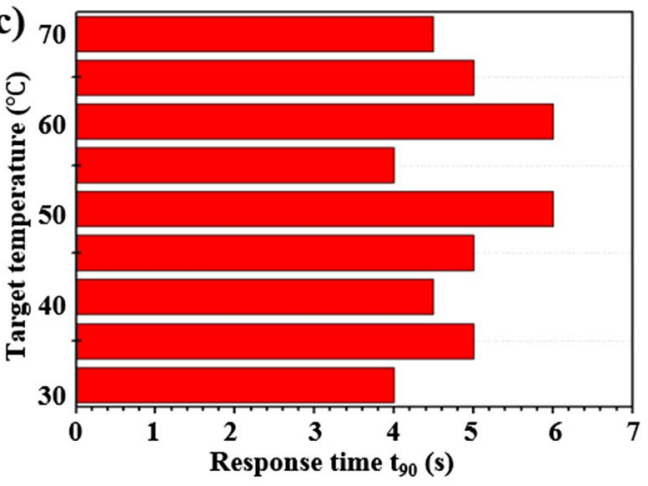

(e)

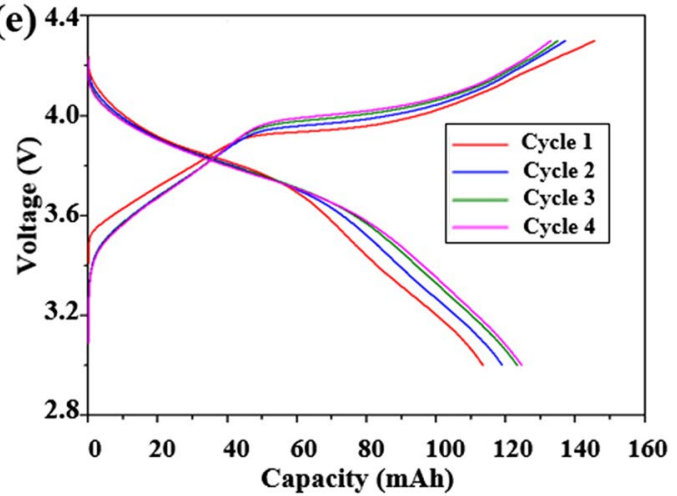

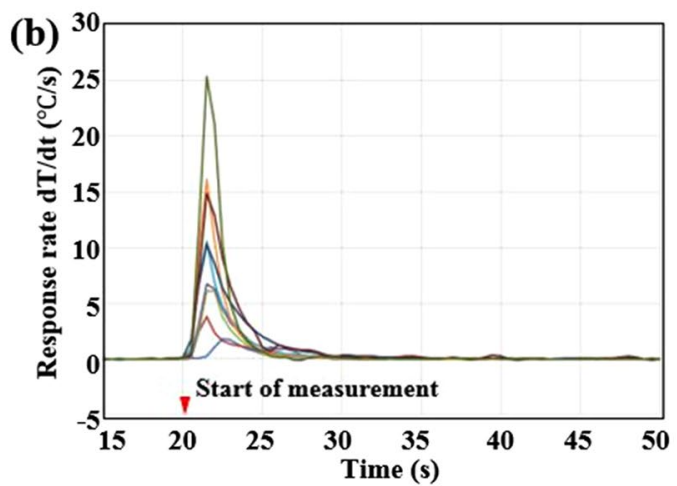

(d)
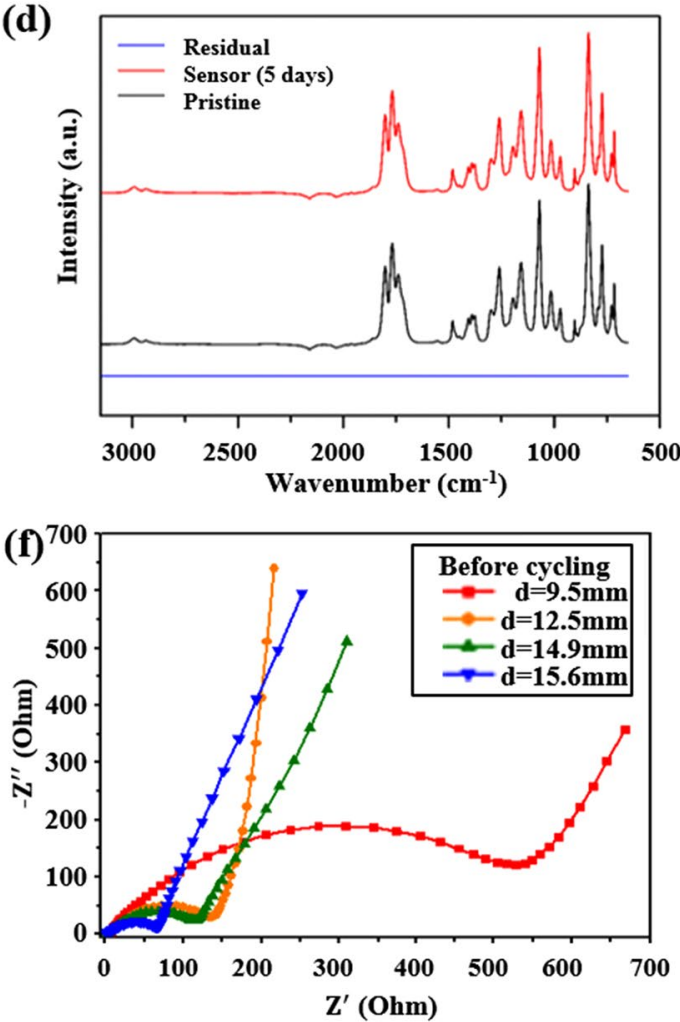

Figure 2. (a) Hot stage temperature measurement with RTD embedded spacers; (b) RTD embedded spacer measurement response rate; (c) RTD embedded spacer measurement response time $t_{90}$; (d) FTIR spectra for electrolyte stability testing; (e) Comparison of charge/discharge curves for sensor embedded cell over vibration; (f) Electrochemical impedance spectroscopy of the constructed cells at OCV (3.1 V vs Li/Li+) with RTD.

discharging, lithiation of the cathode starts at around $3.7 \mathrm{~V}$ without altering the profile of LCO because of the sensor assembly.

Electrochemical impedance spectroscopy (EIS) of the customized CR2032 cells with various cathode diameter $(9.5 \mathrm{~mm}, 12.5 \mathrm{~mm}, 14.9 \mathrm{~mm}, 15.6 \mathrm{~mm}$ ) was conducted at the OCV potential (Fig. 2f). In the high-medium frequency area, the plot depicts depressed semicircles and in the high-frequency area it shows linear Warburg impedance. Ohmic resistances for all the electrodes were similar to each other, $\sim 2.5 \Omega$. Charge transfer resistance differed from each other. An inverse relationship between charge transfer resistance and the size of the electrodes was observed. For the smallest electrode, charge transfer impedance was $527 \Omega$, whereas for the largest electrode, it was $65 \Omega$. The difference in charge transfer resistance between the smallest and largest electrodes arose because the effective area became a significant factor in regions of low frequency, as more charge could pass through the large area, causing impedance to decreas $\mathrm{e}^{24}$. High charge transfer resistance has also been previously reported in coin cells with limited dimension ${ }^{25}$. The impedance of RTD embedded cells was found to be comparable with the other CR2032 coin cells fabricated in a controlled lab environment ${ }^{25,26}$, and the contribution of the customized spacer with an RTD to the cell impedance was limited. Since all four coin cells had limited and comparable Ohmic resistance, the effect of 3D printed spacer on cell performance was negligible and the results obtained from short circuit test of RTD embedded cells could be employed for safety management of ordinary coin cells. 

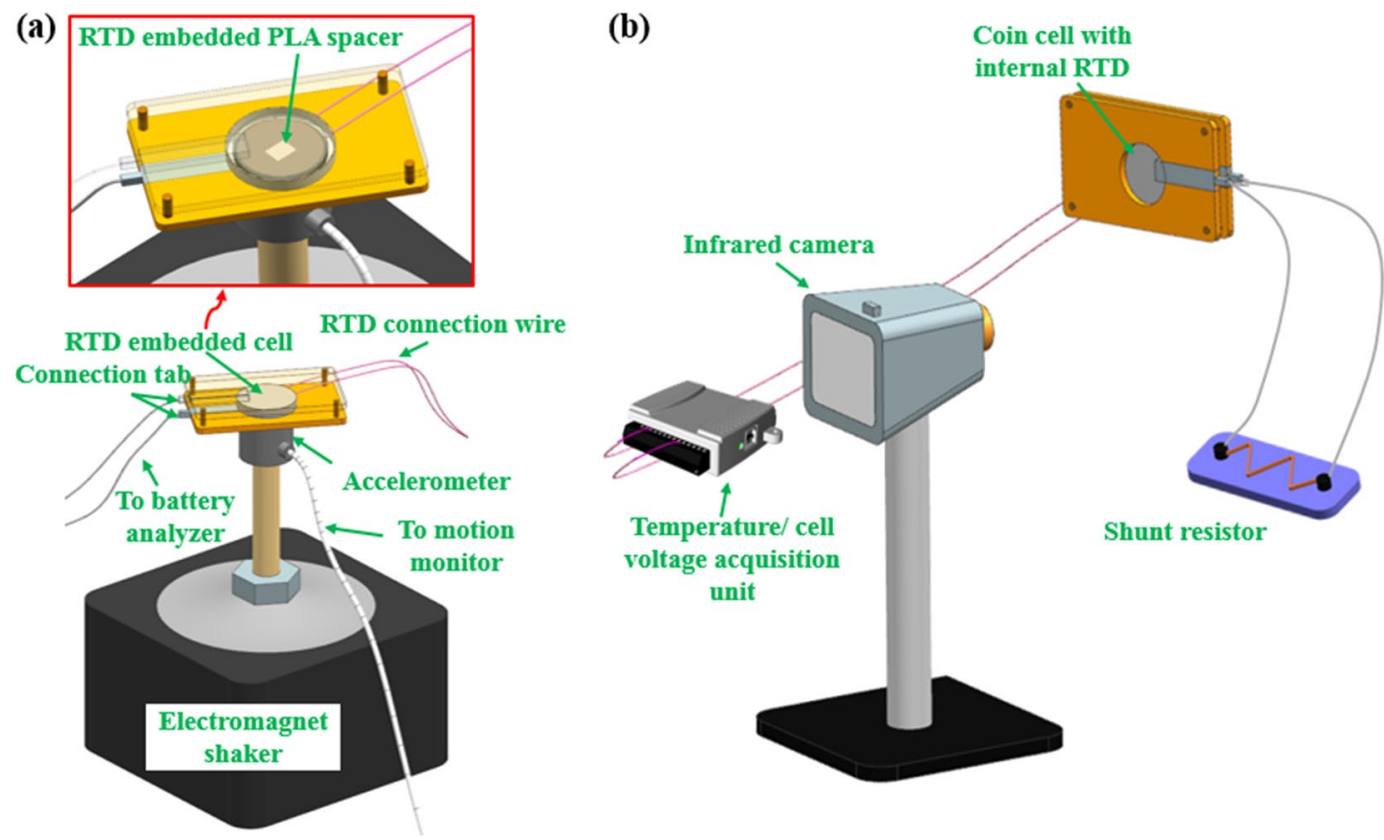

Figure 3. (a) Portable battery cycling setup for LIB performance analysis during vibration; (b) LIB short circuit testing and temperature monitoring platform.

External short circuit test and real-time electrode temperature monitoring. Short circuits are a common concern for aged batteries due to dendrite formation and separator degradation ${ }^{27-29}$, and it is also common in transportation-related accidents such as electric vehicle crashes ${ }^{30,31}$. Short circuits can induce dramatic changes in electrode structure and the electrochemical environment of the battery ${ }^{11}$. An external short circuit test was employed for evaluation of thermal hazard capturing capability of the RTD embedded cells in this work. The platform for external short circuit test and temperature monitoring is shown in Fig. $3 \mathrm{~b}$ with a detailed testing procedure provided in the Method section.

The electrode and battery surface temperature were recorded for the first hour as short circuit related electrochemical reactions were observed to be negligible afterward. An infrared camera (FLIR E40) was used for battery surface temperature recording as a comparison tool for the external RTD measurement result.

A comparison of examined LIB temperature profiles obtained with internal RTD, external RTD, and infrared camera over the first 10 mins of the short circuit test (cathode diameter: $12.5 \mathrm{~mm}$ ) is shown in Fig. 4a. The temperature obtained with the infrared camera is unreliable due to the high-level fluctuation which mainly originates from changes in ambient convection characteristics ${ }^{32}$. In the comparison of RTD measurements, the first difference is in the maximum temperature: $\mathrm{T}_{\max }$ captured by internal RTD is on average $5.8^{\circ} \mathrm{C}$ higher than the external RTD measured average (Fig. 4b). This difference contributes to the thermal contact resistance, which is most significant at electrode-separator contact surface and battery poles ${ }^{33,34}$. The internal RTD measurement avoids the high-level temperature gradient, thus providing accurate electrode temperature monitoring for thermal event detection. The second main difference is in the peak temperature detection time: external RTD detects peak temperature when the internal RTD reading is stabilized or starts decreasing. The measuring efficiency difference arises from energy loss in heat conduction: when heat generated in the electrodes is conducted to the battery surface, part of it is consumed by the temperature rise of battery components, and part of it is dissipated to the air. As a result, the external RTD will fail to reflect the actual temperature rising rate, leaving the cell continuously exposed to potential thermal hazards.

For further evaluation of measurement efficiency, detection time $t_{90}$ is compared for internal and external RTDs. As discussed, $\mathrm{t}_{90}$ is defined as the time to detect $90 \%$ of the maximum temperature rise measured by external RTD $\left(\Delta \mathrm{T}_{\text {ext }}\right) \cdot \Delta \mathrm{T}_{\text {ext }}$ is compared with the maximum temperature rise measured by the internal RTD $\left(\Delta \mathrm{T}_{\text {int }}\right)$ in Fig. 4(b) and with the $\mathrm{t}_{90}$ of two RTDs in Fig. 4c. The internal RTD detects $90 \%$ of $\Delta \mathrm{T}_{\mathrm{EMax}}$ in $7.45 \mathrm{~s}$ on average, which is 7-10 times faster than the external RTD. The measuring efficiency difference builds up with the increase of cathode mass as shown in Fig. 4d, indicating that internal sensor based measurement will be more instructive for thermal hazard detection in LIBs with higher capacity. The high measuring efficiency of the internal RTD is attributed to the well maintained sensor-electrode contact. Reliable sensor-electrode contact is maintained by the pressure load applied during cell fabrication, allowing for effective heat conduction from the current collector to internal RTD. In existing work on electrode temperature measurement, the sensors were directly applied onto the porous electrode material ${ }^{8}$ with limited control of sensor-electrode contact and installation stress. Excessive installation pressure could lead to electrode material damage and impair battery performance ${ }^{35}$. Also, electrode particle cracking and peel-off occurred during LIB thermal runaway, which further impairs the unsecured sensor-electrode contact and can be observed in SEM images acquired on the cathode before and after the short circuit test, as shown in Figs. 4e,f. 

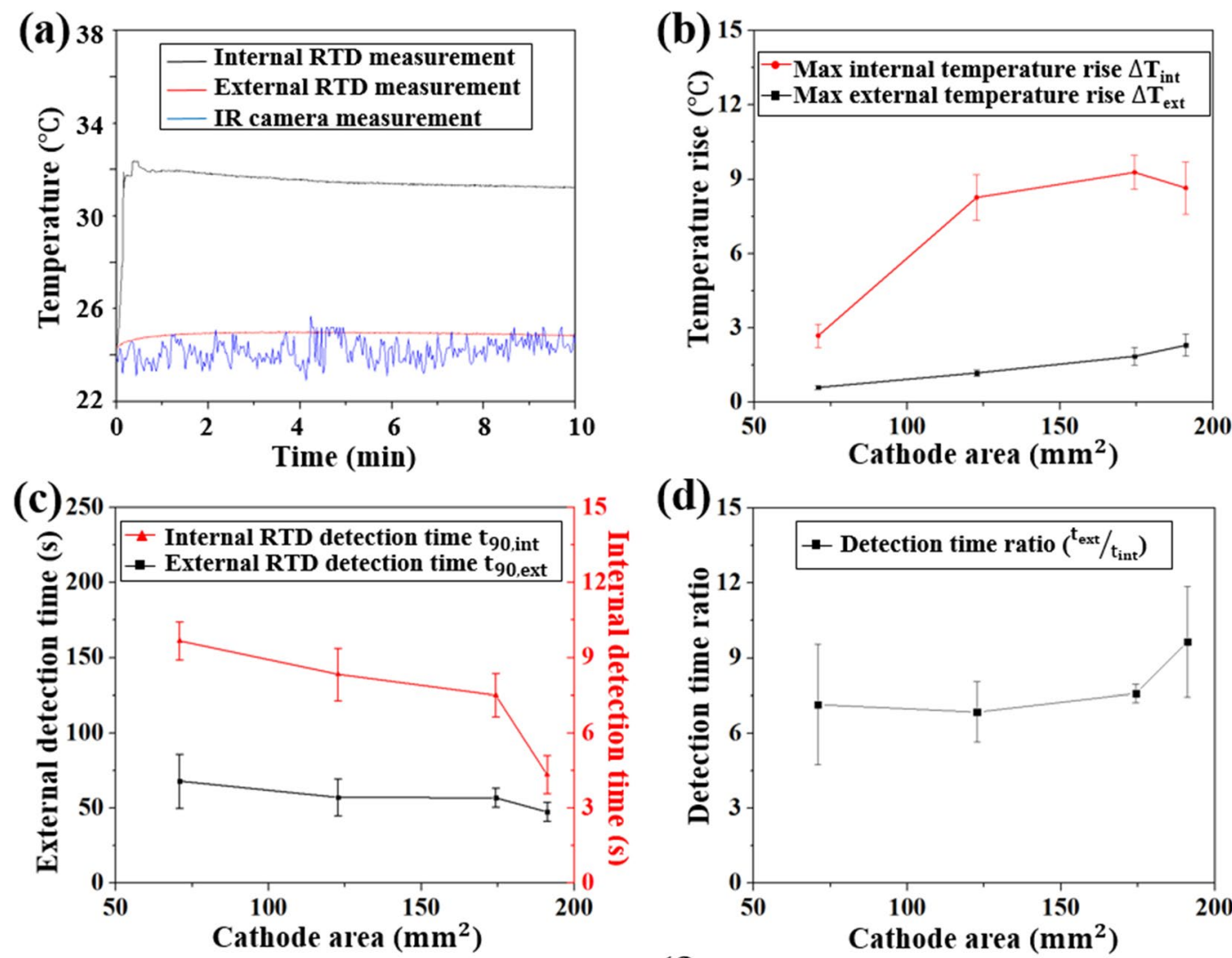

(e)
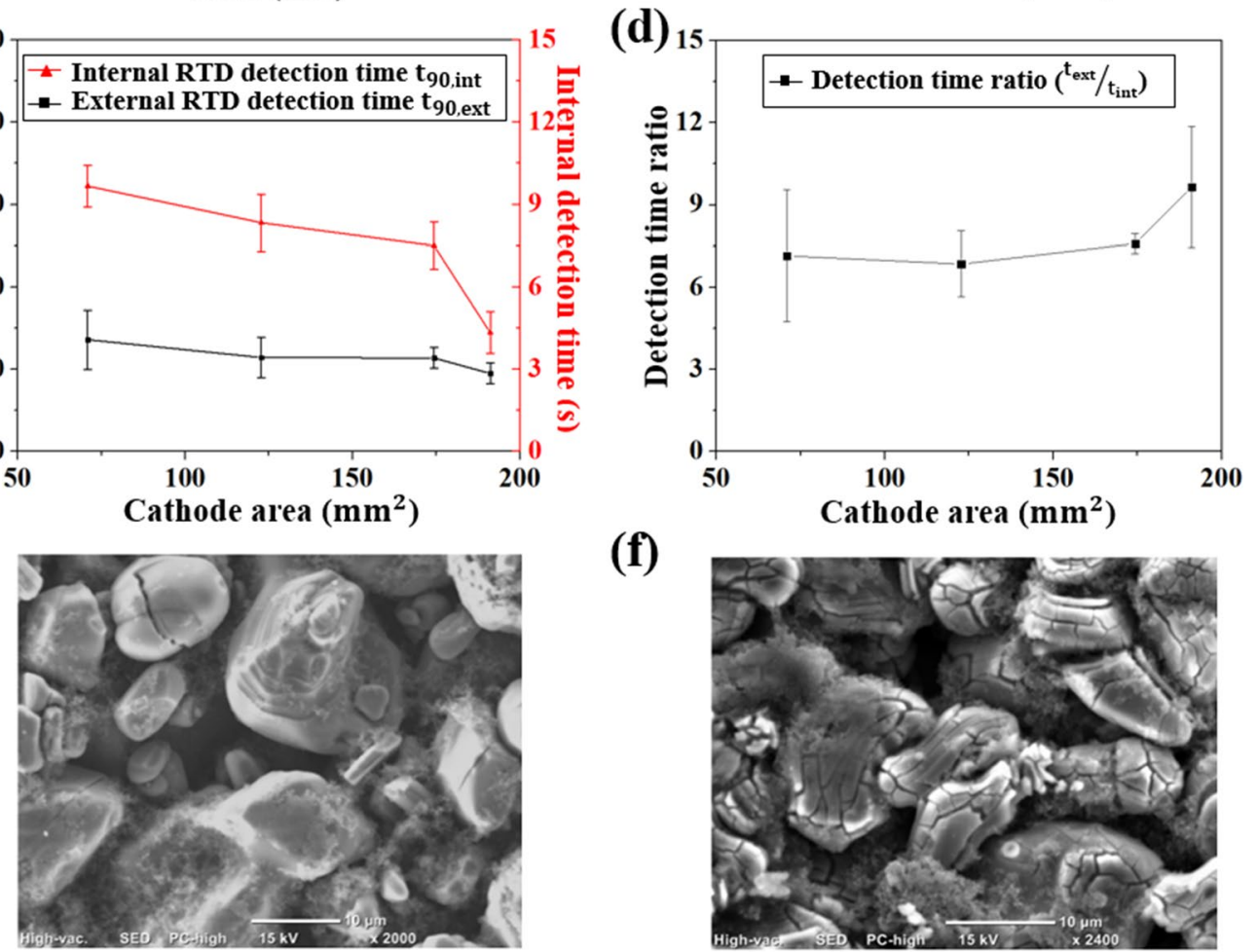

(f)

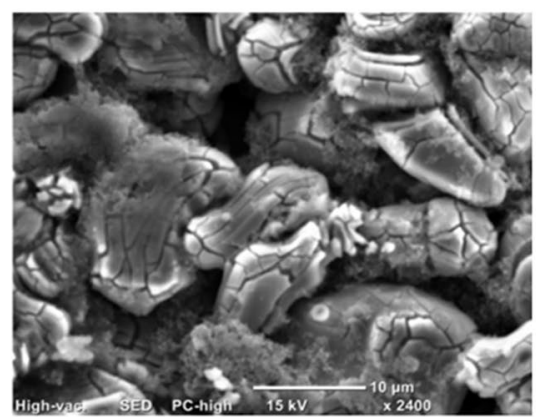

Figure 4. (a) Temperature measurements with internal RTD, external RTD and infrared camera in the short circuit test; (b) Maximum temperature rise detected by internal and external RTDs; (c) RTD detection time $t_{90 \text {, int }}$ and $t_{90, \text { ext }}$ of internal and external RTDs; (d) Detection time ratio $t_{\text {ext }} / t_{\text {int }} ;$ (e) SEM image of LCO cathode before short circuit test and (f) after short circuit test.

Despite electrode temperature monitoring with improved efficiency and accuracy, an internal RTD can also be used for the prediction of cell temperature evolution after the detected thermal hazard. When high temperature is detected in a LIB pack, involved cells will be removed from the circuit ${ }^{36}$ and the subsequent cooling process is typically accomplished by convection in $\operatorname{air}^{32}$. As the current drains rapidly in the short circuit test (within $5 \mathrm{~s}$ ), the temperature decrease process of CR 2023 coin cells can be modeled with natural convection, and a general energy balance equation of LIB cells can be written $\mathrm{as}^{32}$ :

$$
\mathrm{mC} \frac{\mathrm{dT}_{\mathrm{ext}}}{\mathrm{dt}}=\dot{\mathrm{Q}}-\mathrm{hA}\left(\mathrm{T}_{\mathrm{ext}}-\mathrm{T}_{\infty}\right)
$$

where $\mathrm{m}$ is cell mass, $\mathrm{C}$ is heat capacity of the cell, $\mathrm{h}$ is convective heat transfer coefficient, $\mathrm{A}$ is convection area, $\mathrm{T}_{\infty}$ is ambient temperature, $\mathrm{T}_{\text {ext }}$ is battery surface temperature and $\mathrm{Q}$ is the internal heat source term. A simple way to obtain the analytical solution of Eq. (1) is to assume uniform temperature distribution of the cell and neglect the $\mathrm{Q}$ term during the cooling process, which provides a solution for $\mathrm{T}_{\mathrm{ext}} \mathrm{as}^{32}$ :

$$
\left(\mathrm{T}_{\text {ext }}-\mathrm{T}_{\infty}\right)=\left(\mathrm{T}_{\mathrm{e} 0}-\mathrm{T}_{\infty}\right) \exp \left(-\frac{\mathrm{hA}}{\mathrm{mc}} \mathrm{t}\right)=\left(\mathrm{T}_{\mathrm{e} 0}-\mathrm{T}_{\infty}\right) \exp \left(-\frac{\mathrm{t}}{\tau}\right)
$$


with $\mathrm{T}_{\mathrm{ext}}=\mathrm{T}_{\mathrm{e} 0}$ at the beginning of convective cooling and $\tau$ is the convection time constant $\left(\frac{\mathrm{hA}}{\mathrm{mc}}\right)$ that can be determined with temperature evolution in natural convection cooling of the cell. However, our previous measurements show that there is a significant temperature gradient within the cell, and the thermal conduction from the electrode to the cell surface is non-negligible at the start of the cooling process. Thus, the model in Eq. (2) will fail to reflect the actual change of battery surface temperature and it is necessary to consider the thermal energy transferred from the electrode for accurate surface temperature prediction. Considering this, the cooling process is separated into two phases: in the first time period the electrode temperature is different from the cell surface temperature, and the electrode system provides the internal heat source term $\dot{Q}$, in the second phase cell temperature is relatively uniform and Q can be neglected. Based on the measurement efficiency comparison of internal and external RTD in Fig. 4(d), separation for periods 1 and 2 can be set at $5 t_{i n}$, where $t_{\text {in }}$ is the time for the internal RTD to detect the maximum electrode temperature, as shown in Fig. 5(a). The new energy balance equation for the battery can be written as:

$$
\begin{gathered}
\left\{\mathrm{mC} \frac{\mathrm{dT}_{\text {ext }}}{\mathrm{dt}}=\dot{\mathrm{Q}}-\mathrm{hA}\left(\mathrm{T}_{\text {ext }}-\mathrm{T}_{\infty}\right) \mathrm{t}_{\text {in }} \leq \mathrm{t}<5 \mathrm{t}_{\text {in }}\right. \\
\left\{\mathrm{mC} \frac{\mathrm{dT}_{\text {ext }}}{\mathrm{dt}}=-\mathrm{hA}\left(\mathrm{T}_{\text {ext }}-\mathrm{T}_{\infty}\right) 5 \mathrm{t}_{\text {in }} \leq \mathrm{t}\right.
\end{gathered}
$$

The internal heat source term $\dot{Q}$ is given as:

$$
\dot{\mathrm{Q}}=-\mathrm{m}_{\mathrm{e}} \mathrm{C}_{\mathrm{e}} \frac{\mathrm{dT}_{\mathrm{in}}}{\mathrm{dt}}
$$

where $\mathrm{T}_{\text {in }}$ is the temperature obtained with internal RTD, $\mathrm{m}_{\mathrm{e}}$ and $\mathrm{C}_{\mathrm{e}}$ are the mass and heat capacity of the system that possesses a temperature of $\mathrm{T}_{\mathrm{in}}$. It is assumed that for the $\mathrm{LiCoO}_{2}$ cathode, the $\mathrm{Li}$ anode and stainless-steel spacer above the anode possess a temperature of $\mathrm{T}_{\mathrm{in}}$, considering the tight contact between these layers, and the values of $\mathrm{m}_{\mathrm{e}}$ and $\mathrm{C}_{\mathrm{e}}$ are calculated, accordingly ${ }^{37,38}$. After reaching the maximum value at $\mathrm{t}_{\mathrm{in}}, \mathrm{T}_{\mathrm{in}}$ is modeled to decay exponentially:

$$
\mathrm{T}_{\mathrm{in}}=\mathrm{T}_{\infty}+\left(\mathrm{T}_{\mathrm{i} 0}-\mathrm{T}_{\infty}\right) \cdot \exp (-\mathrm{a} \cdot \mathrm{t})
$$

where $\mathrm{a}$ is the time coefficient to be determined and the value of $\mathrm{T}_{\text {in }}$ over $\mathrm{t}_{\mathrm{in}} \leq \mathrm{t} \leq 2 \mathrm{t}_{\text {in }}$ is used for the derivation of a. Then the solution of Eq. (3a) can be obtained numerically with MATLAB providing the initial value of $\mathrm{T}_{\text {ext }}$ at $t=\mathrm{t}_{\mathrm{in}}$, and the solution for Eq. (3b) is provided by Eq. (2). A comparison of battery surface temperature predictions with and without the contribution of the internal heat source $\dot{Q}$ is shown in Fig. 5(b). The new model reduces error in maximum surface temperature prediction as shown in Fig. $5 c$. The ratio of $\mathrm{T}_{\mathrm{EMax}}$ prediction error remains steady over different cathode sizes and the model can be applied to cells with higher electrode mass. The proposed model can also predict battery temperature evolution after thermal hazard detection and avoid thermal hazard after removal of the cell.

The relation between temperatures measured with internal and external RTDs can also be used for improving the efficiency of surface temperature-based battery thermal runaway detection. A curved surface correlating maximum surface temperature increasing rate with cathode area, $A$, and cathode temperature increase, $\Delta T_{\text {int }}$, in short circuit is plotted by biharmonic spline interpolation and $4^{\text {th }}$ order polynomial fitting with MATLAB in Fig. 5e. The relation can be written as:

$$
\begin{aligned}
\left(\frac{d T_{\text {ext }}}{d t}\right)_{\max }= & -1.83+0.060 \cdot A+0.36 \cdot \Delta T_{\text {int }}-6.88 \cdot 10^{-4} \cdot A^{2}-7.17 \cdot 10^{-3} \\
& \cdot A \cdot \Delta T_{i n t}-0.011 \cdot \Delta T_{i n t}^{2}+3.28 \cdot 10^{-6} \cdot A^{3}+4.50 \cdot 10^{-5} \cdot A^{2} \\
& \cdot \Delta T_{i n t}+2.14 \cdot 10^{-4} \cdot A \cdot \Delta T_{i n t}^{2}-5.77 \cdot 10^{-9} \cdot A^{4}-7.84 \cdot 10^{-8} \\
& \cdot A^{3} \cdot \Delta T_{i n t}-8.83 \cdot 10^{-7} \cdot A^{2} \cdot \Delta T_{i n t}^{2}
\end{aligned}
$$

The relation between $\Delta T_{\text {int }}$ and cathode area, $A$, of a coin cell is fitted with a cubic polynomial function:

$$
\Delta T_{\text {int }}=-21.364+3.874 \cdot A-0.164 \cdot A^{2}+2.301 \cdot 10^{-3} \cdot A^{3}
$$

It is found from Eq. (6) that the increasing rate of battery surface temperature in short circuit related thermal runaway is dependent on both electrode temperature rise and electrode mass. This increase arises because exothermic reaction in short circuit is mainly composed of SEI decomposition, reaction between cathode, anode and electrolyte and electrolyte decomposition at the electrolyte-electrode interface [1], and these reactions are dependent on electrochemically active mass as shown in Eq. (7). Electrode surface area determines the rate of thermal energy transfer from the electrode to battery surface. Thus, the thermal runaway risk level cannot be simply predicted with the change in surface temperature rise, but the cell capacity also needs to be taken into consideration. For CR 2032 cell with specific LCO cathode mass, when the increasing rate of surface temperature approaches the top part of the curved surface in Fig. 6e, there will be a high risk of thermal runaway and effective cooling such as forced air cooling should be applied to control the electrode temperature and detrimental thermal gradient across the cell ${ }^{39}$. For similar increasing rate of surface temperature, batteries with lower electrode mass 

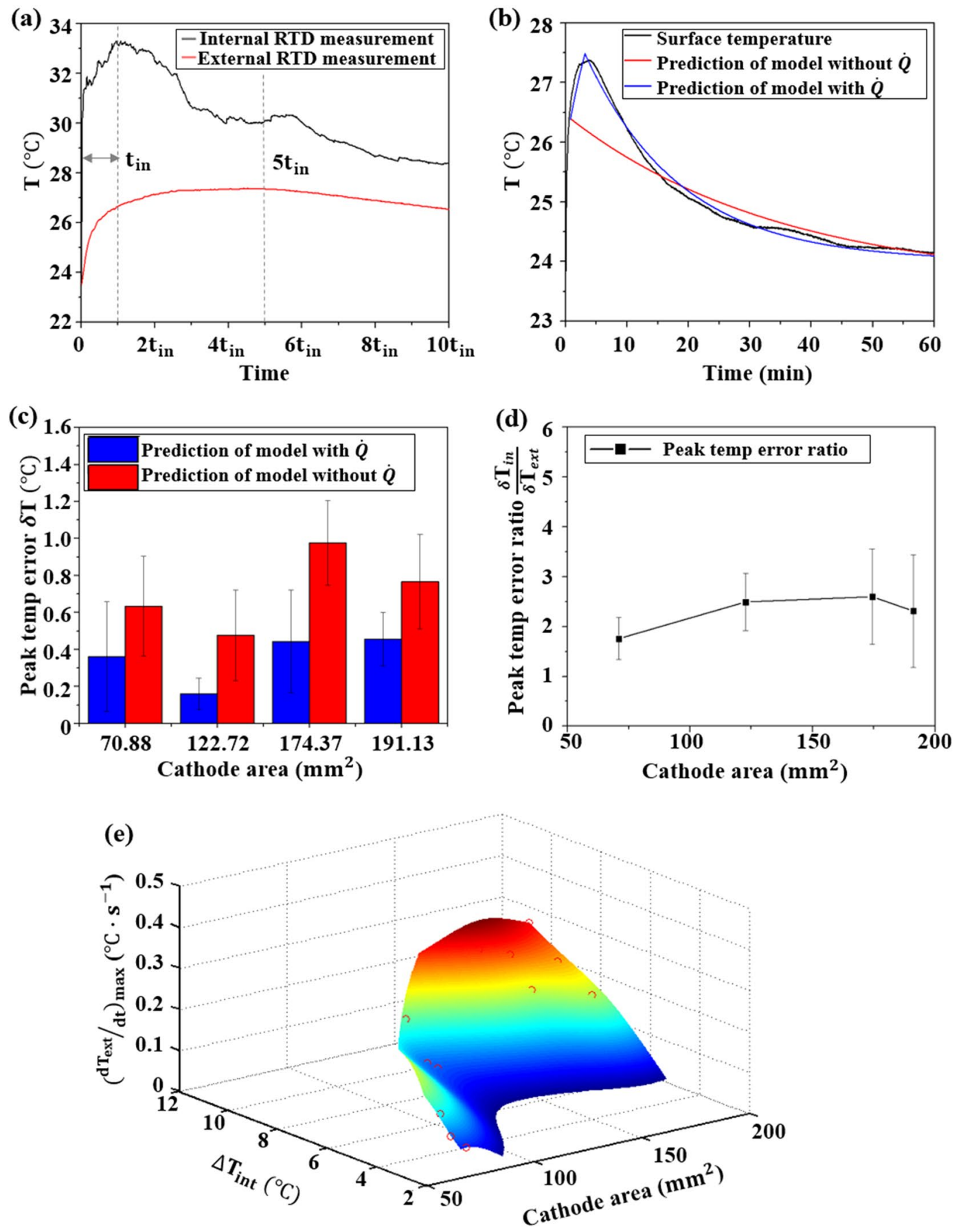

Figure 5. (a) Two phases of heat transfer in battery short circuit test; (b) Representative comparison of temperature model predictions; (c) Peak temperature prediction error comparison; (d) Peak temperature prediction error ratio; (e) Relation between cathode mass, peak internal temperature and maximum battery surface temperature rising rate.

will be more prone to thermal hazards, and a cell capacity dependent safety temperature threshold can be determined based on external RTD measurement with the relation between internal and external RTD reading established. Besides, some fluctuation was observed in temperature measured by internal RTD during the short circuit event. It represented instability in local heat generation and transfer. The fluctuation originated from changes in LIB structure during short circuit, including lithium dendrite formation, current collector dissolution, electrode particle delamination, gas generation, etc. Dendrite could reduce cell resistance and trigger internal shorting ${ }^{27}$, which could enhance the short circuit. Current collector dissolution and gas generation influenced cell impedance and local heat transfer condition ${ }^{40,41}$. Electrode particle could delaminate during thermal runaway ${ }^{42}$, leading to abrupt drop in cell capacity and heat generation rate at the region of delamination. Current collector dissolution and electrode particle delamination observed in short circuit test can be found in Supplementary Fig. S1. The 


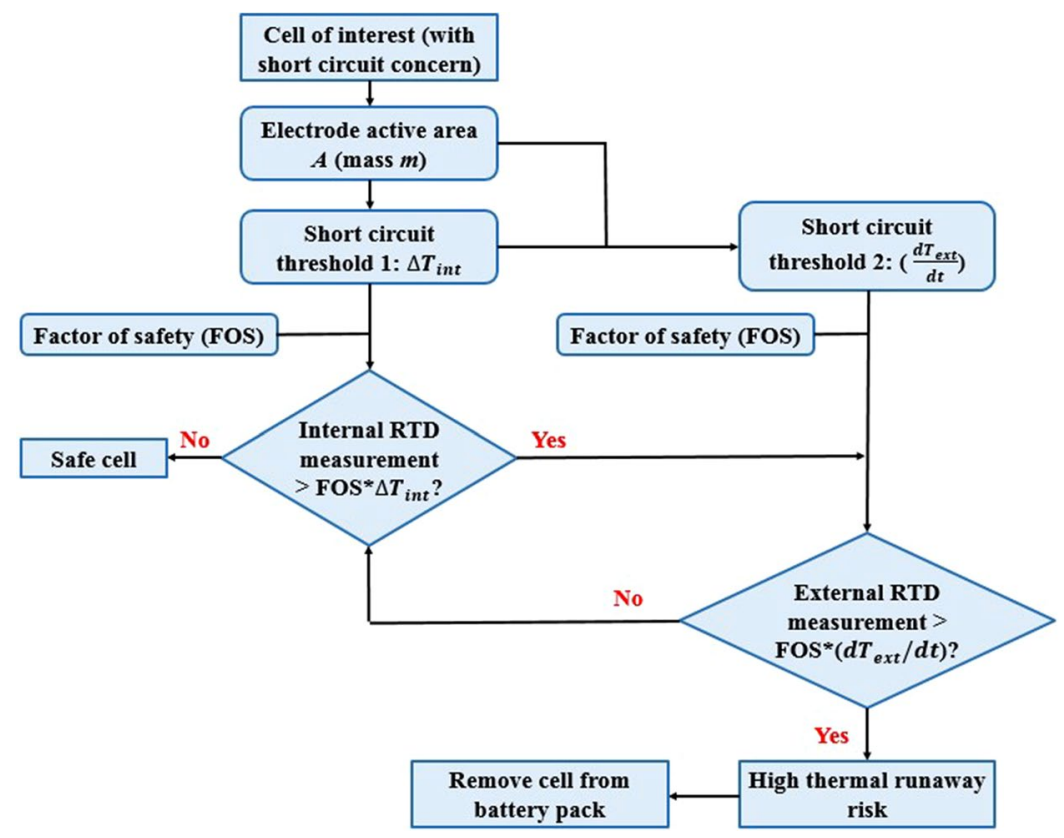

Figure 6. Strategy for protecting coin cell against short circuit related thermal runaway.

local measurement capability of internal RTD captured these transient regional processes, and it was instructive for LIB thermal runaway detection and prevention.

For prevention of short circuit related thermal runaway, a thermal runaway risk assessing strategy is developed for CR2032 coin cell based on internal and external RTD measurements as well as established relations between the electrode and battery surface temperature from Eqs ( 6 and 7):

This thermal runaway prevention strategy consists of internal RTD and external RTD based protection. Due to the superior measuring efficiency, internal RTD measurement is critical when short circuit is of concern for a cell. Electrode temperature rise, $\Delta T_{\text {int }}$, is used as the early signature of thermal runaway and if the measured value excesses range for safe battery operation, the increasing rate of battery surface temperature will be calculated with the external RTD and employed for verification of assessment. If the battery surface temperature is rising in an unsafe manner based on Eq. (6), a high risk for thermal runaway is identified for the analyzed cell and it should be disconnected immediately. Scientific experimental efforts are underway to test multiple RTD sensors in pouch full-cell configurations for local heat generation mapping of the electrode.

\section{Conclusion}

In this study, a RTD sensor embedded PLA spacer was developed and incorporated inside the CR2032 coin cells for in-operando temperature monitoring. The customized cell presented reliable sensor-electrode contact and high structural robustness during cycling over continuous vibration, based on U.S. Navy standards. In the short circuit test, the internal RTD provided superior performance in measuring the electrode temperature compared to the external RTD. In addition, the internal RTD reported peak temperature 7-10 times faster than the external RTD, providing a better chance for battery hazard prevention. Electrode temperature measurement with an internal RTD was also employed for battery surface temperature prediction in short circuit, providing surface temperature evolution prediction with reduced error. Finally, the relation between internal RTD measurement, external RTD measurement and electrode mass was obtained for reliable short circuit detection and prediction based on battery surface temperature measurement.

\section{Methods}

RTD sensor embedded lithium-ion coin cell fabrication. To develop a feasible approach to detect battery thermal runaway in-operando and meet requirement on commercial LIBs, the design of a customized RTD embedded LIB cell was dictated by three key factors: (1) to acquire the cathode electrode temperature accurately and effectively; (2) to eliminate sensor induced interference due to LIB operation; and (3) to minimize sensor induced electrode damage. For accurate real-time electrode temperature monitoring, reliable sensor-electrode contact is desired, which requires that the sensor surface pairs well with the electrode. Pt1000 RTD (by Omega Engineering Inc.) with a $4 \mathrm{~mm} \times 5 \mathrm{~mm}$ flat $\mathrm{Al}_{2} \mathrm{O}_{3}$ sensing surface was selected in this work. Pt1000 RTD has been extensively applied in process temperature monitoring, including LIB electrolyte stability analysis ${ }^{43,44}$, and LIB cycling $^{45}$. The Pt sensing element employed in this work had a temperature dependent resistance, $\mathrm{R}$, of:

$$
\mathrm{R}=\left(1+3.9083 \cdot 10^{-3 \circ} \mathrm{C}^{-1} \cdot \mathrm{T}-5.77510^{-7{ }^{\circ}} \mathrm{C}^{-2} \cdot \mathrm{T}^{2}-4.18310^{-12 \circ} \mathrm{C}^{-4} \cdot \mathrm{a} \cdot \mathrm{T}^{3}\right) \mathrm{k} \Omega
$$

where $\mathrm{a}=(\mathrm{T}-100)^{\circ} \mathrm{C}$ for $\mathrm{T}<0{ }^{\circ} \mathrm{C}$ and $\mathrm{a}=0^{\circ} \mathrm{C}$ for $\mathrm{T}>0{ }^{\circ} \mathrm{C}$. 
The RTD provided an average sensitivity of $3.883 \Omega /{ }^{\circ} \mathrm{C}$ within the ordinary battery operation temperature of $-10^{\circ} \mathrm{C}$ to $50^{\circ} \mathrm{C}^{46}$.

A strip of aluminum current collector was applied across the PLA spacer for cell conductivity. By embedding the customized spacer in the CR2032 cell, a new pattern of measuring electrode temperature from the electrode current collector was achieved, which eliminated sensor induced disturbance to battery operation by removing the sensor from the gap between the electrodes. Thickness variation of the RTD embedded spacer was controlled within $10 \mu \mathrm{m}$ for contact reliability between the RTD and the electrode. The well-maintained spacer-electrode pairing can improve measurement efficiency and control electrode damage during temperature monitoring. In addition to application in CR 2032 coin cell shown in Fig. 1, 3D printing technique provided visibility for the RTD embedded spacer to be applied in commercial LIBs, including 18650 cell. With customized geometry design, the RTD can be applied to measure electrode temperature at different locations of the jelly roll. Illustration of RTD application in 18650 cell is provided in Supplementary Fig. S2.

RTD embedded spacer measuring efficiency, accuracy and electrochemical stability assessment. In order to ensure that the RTD embedded PLA spacer is electrochemically inert and will not introduce side reactions during LIB operation, the spacer was submerged in LIB electrolyte $\left(1 \mathrm{M} \mathrm{LiPF}_{6} \mathrm{EC}_{\mathrm{DEC}}\right.$ (Sigma-Aldrich)) for 5 days. A FTIR spectrum of the electrolyte was acquired and compared with the spectrum of the pristine electrolyte. For temperature measurement efficiency and accuracy evaluation, the RTD embedded spacer was firstly applied on a PID controlled hot stage, which was preset to various temperatures ranging from $30^{\circ} \mathrm{C}$ to $70^{\circ} \mathrm{C}$. The spacer was mounted onto the hot stage with a pressure of $1500 \mathrm{psi}$ (same pressure applied in cell crimping), while temperature readings from the RTD were recorded for 3 mins. Then, the CR2032 coin cell with RTD embedded spacer was cycled under vibration loading condition, as described in NAVSEA $9310^{47}$ and depicted in Fig. 3a. Four electrochemical cycles were completed and the electrochemical performance of the cell was analyzed.

Short circuit testing with RTD embedded coin cell. In the short circuit test, a $15 \mathrm{~m} \Omega$ shunt resistor was used for external shorting, and the total resistance of the external circuit was determined to be $19.8 \mathrm{~m} \Omega$. The low resistance external circuit generated a "hard" short circuit condition per NAVSEA $9310^{47}$. Four groups of RTD embedded LIBs with LCO cathode diameters of $9.5 \mathrm{~mm}, 12.5 \mathrm{~mm}, 14.9 \mathrm{~mm}$, and $15.6 \mathrm{~mm}$ were prepared (LCO loading $14.53 \mathrm{mg} / \mathrm{cm}^{2}$ ) and analyzed in the short circuit test. Before testing, the cells were cycled between $3.0 \mathrm{~V}$ and $4.3 \mathrm{~V}$ for cathode electrolyte interface (CEI) formation ${ }^{23}$, which would decompose during the short circuit test and introduce further exothermic reaction ${ }^{48}$. The cells were then fully charged and shorted for 24 hours according to NAVSEA $9310^{47}$. Two RTDs were used for electrode and battery surface temperature measurement: one embedded on the spacer (internal RTD) and one attached on the battery surface (external RTD).

\section{Data Availability}

The datasets generated during and analyzed during the current study are available from the corresponding author on reasonable request.

\section{References}

1. Lee, J. et al. Unlocking the potential of cation-disordered oxides for rechargeable lithium batteries. Science 343, 519-522 (2014).

2. Wang, C.-Y. et al. Lithium-ion battery structure that self-heats at low temperatures. Nature 529, 515-518 (2016).

3. Wu, H., Zhuo, D., Kong, D. \& Cui, Y. Improving battery safety by early detection of internal shorting with a bifunctional separator. Nature Communications 5, 5193 (2014).

4. Chen, Z., Xiong, R., Lu, J. \& Li, X. Temperature rise prediction of lithium-ion battery suffering external short circuit for all-climate electric vehicles application. Applied Energy 213, 375-383 (2018).

5. Peng, P. \& Jiang, F. Thermal safety of lithium-ion batteries with various cathode materials: A numerical study. International Journal of Heat and Mass Transfer 103, 1008-1016 (2016).

6. Wu, M.-S., Chiang, P.-C. J., Lin, J.-C. \& Jan, Y.-S. Correlation between electrochemical characteristics and thermal stability of advanced lithium-ion batteries in abuse tests-short-circuit tests. Electrochimica Acta 49, 1803-1812 (2004).

7. Abaza, A. et al. Experimental study of internal and external short circuits of commercial automotive pouch lithium-ion cells. Journal of Energy Storage 16, 211-217 (2018).

8. Zhang, G. et al. Reaction temperature sensing (RTS)-based control for Li-ion battery safety. Scientific Reports 5, 18237 (2015).

9. Finegan, D. P. et al. Characterising thermal runaway within lithium-ion cells by inducing and monitoring internal short circuits. Energy \& Environmental Science 10, 1377-1388 (2017).

10. Liu, B. et al. Safety issues caused by internal short circuits in lithium-ion batteries. Journal of Materials Chemistry A 6, 21475-21484 (2018).

11. Zhao, R., Liu, J. \& Gu, J. Simulation and experimental study on lithium ion battery short circuit. Applied Energy 173, 29-39 (2016).

12. Zhang, M. et al. Internal short circuit trigger method for lithium-ion battery based on shape memory alloy. Journal of The Electrochemical Society 164, A3038-A3044 (2017).

13. Novais, S. et al. Internal and external temperature monitoring of a Li-ion battery with fiber bragg grating sensors. Sensors 16, 1394 (2016).

14. Liu, B., Yin, S. \& Xu, J. Integrated computation model of lithium-ion battery subject to nail penetration. Applied Energy 183, 278-289 (2016).

15. Orendorff, C. J., Roth, E. P. \& Nagasubramanian, G. Experimental triggers for internal short circuits in lithium-ion cells. Journal of Power Sources 196, 6554-6558 (2011).

16. Tomar, V. Insights into the effects of tensile and compressive loadings on microstructure dependent fracture of trabecular bone. Engineering Fracture Mechanics 76, 884-897 (2009).

17. Lee, S.-M., Kim, J.-Y. \& Byeon, J.-W. Failure analysis of short-circuited lithium-ion battery with nickel-manganese-cobalt/graphite electrode. Journal of Nanoscience and Nanotechnology 18, 6427-6430 (2018).

18. Tomar, V., Gan, M. \& Kim, H. S. Atomistic analyses of the effect of temperature and morphology on mechanical strength of Si-C-N and Si-C-O nanocomposites. Journal of the European Ceramic Society 30, 2223-2237 (2010). 
19. Li, B. et al. Investigation of response of LiCoO2 cathode to dynamic impact using Raman imaging-based analyses. JOM 70, $1423-1429$ (2018).

20. Lin, X., Wang, W., Li, B., Miao, J. \& Liu, Y. Research into thermal response time fault diagnosis of temperature sensor in high-speed EMU. In International Conference on Electrical and Information Technologies for Rail Transportation. 475-482 (Springer, 2017).

21. Wang, Q., Sun, J., Yao, X. \& Chen, C. Thermal stability of LiPF6/EC+ DEC electrolyte with charged electrodes for lithium ion batteries. Thermochimica Acta 437, 12-16 (2005).

22. Ellis, L. et al. A new method for determining the concentration of electrolyte components in lithium-ion cells, using fourier transform infrared spectroscopy and machine learning. Journal of The Electrochemical Society 165, A256-A262 (2018).

23. Jurng, S., Brown, Z. L., Kim, J. \& Lucht, B. L. Effect of electrolyte on the nanostructure of the solid electrolyte interphase (SEI) and performance of lithium metal anodes. Energy \& Environmental Science 11, 2600-2608 (2018).

24. Ahmed, R. \& Reifsnider, K. Study of influence of electrode geometry on impedance spectroscopy. Int. J. Electrochem. Sci 6, $1159-1174$ (2011).

25. Adams, R. A. et al. Binder-free N-and O-rich carbon nanofiber anodes for long cycle life K-ion batteries. ACS Applied Materials \& Interfaces $9,17872-17881$ (2017).

26. Juarez-Robles, D., Chen, C.-F., Barsukov, Y. \& Mukherjee, P. P. Impedance evolution characteristics in lithium-ion batteries. Journal of The Electrochemical Society 164, A837-A847 (2017).

27. Love, C. T., Baturina, O. A. \& Swider-Lyons, K. E. Observation of lithium dendrites at ambient temperature and below. ECS Electrochemistry Letters 4, A24-A27 (2015).

28. Han, F. et al. High electronic conductivity as the origin of lithium dendrite formation within solid electrolytes. Nature Energy 4 , $187-196(2019)$.

29. Lagadec, M. F., Zahn, R. \& Wood, V. Characterization and performance evaluation of lithium-ion battery separators. Nature Energy 4, 16-25 (2018)

30. National Transportation Safety Board. Car with automated vehicle controls crashes into roadway barrier, https://www.ntsb.gov/ investigations/Pages/HWY18FH011.aspx (2018).

31. National Transportation Safety Board. Collision with postcrash fire involving an electric passenger vehicle, https://www.ntsb.gov/ investigations/Pages/HWY18FH013.aspx (2018).

32. Mistry, A. N., Palle, H. R. \& Mukherjee, P. P. In operando thermal signature probe for lithium-ion batteries. Applied Physics Letters 114, 023901 (2019).

33. Yi, J., Kim, U. S., Shin, C. B., Han, T. \& Park, S. Three-dimensional thermal modeling of a lithium-ion battery considering the combined effects of the electrical and thermal contact resistances between current collecting tab and lead wire. Journal of the Electrochemical Society 160, A437-A443 (2013).

34. Chen, S., Wan, C. \& Wang, Y. Thermal analysis of lithium-ion batteries. Journal of power sources 140, 111-124 (2005)

35. Liu, D. et al. On the stress characteristics of graphite anode in commercial pouch lithium-ion battery. Journal of Power Sources 232, 29-33 (2013).

36. Lee, B. H. \& Kim, S. W. Development of battery management system for nickel-metal hydride batteries in electric vehicle applications. Journal of Power Sources 109, 1-10 (2002).

37. Kawaji, H. et al. Low temperature heat capacity and thermodynamic functions of LiCoO 2. Journal of Thermal Analysis and Calorimetry 68, 833-839 (2002).

38. CRC. CRC Handbook of Chemistry and Physics: A Ready-reference Book of Chemical and Physical Data (ed. Lide, D. R.) 4-123 (CRC Press, 1995).

39. Carter, R. \& Love, C. T. Modulation of lithium plating in Li-Ion batteries with external thermal gradient. ACS Applied Materials \& Interfaces 10, 26328-26334 (2018).

40. Wang, Q. et al. Thermal runaway caused fire and explosion of lithium ion battery. Journal of power sources 208, 210-224 (2012).

41. Fear, C., Juarez-Robles, D., Jeevarajan, J. A. \& Mukherjee, P. P. Elucidating copper dissolution phenomenon in Li-Ion cells under overdischarge extremes. Journal of The Electrochemical Society 165, A1639-A1647 (2018).

42. Yokoshima, T. et al. Operando analysis of thermal runaway in lithium ion battery during nail-penetration test using an $\mathrm{x}$-ray inspection system. Journal of The Electrochemical Society 166, A1243-A1250 (2019).

43. Logan, E. et al. A study of the physical properties of Li-Ion battery electrolytes containing esters. Journal of The Electrochemical Society 165, A21-A30 (2018).

44. Keil, P., Rumpf, K. \& Jossen, A. Thermal impedance spectroscopy for Li-ion batteries with an IR temperature sensor system. In 2013 World Electric Vehicle Symposium and Exhibition (EVS27). 1-11 (IEEE, 2013).

45. Wang, P. et al. Real-time monitoring of internal temperature evolution of the lithium-ion coin cell battery during the charge and discharge process. Extreme Mechanics Letters 9, 459-466 (2016).

46. Aris, A. M. \& Shabani, B. An Experimental study of a lithium ion cell operation at low temperature conditions. Energy Procedia 110, 128-135 (2017).

47. Naval Ordnance Safety and Security Activity. NAVSEA S9310-AQ-SAF-010 Technical manual for navy lithium battery safety program responsibilities and procedures, 2nd revision, http://www.public.navy.mil/NAVSAFECEN/Documents/afloat/Surface/CS/ Lithium_Batteries_Info/LithBatt_NAVSEA_TMS9310.pdf (2010).

48. Bandhauer, T. M., Garimella, S. \& Fuller, T. F. A critical review of thermal issues in lithium-ion batteries. Journal of the Electrochemical Society 158, R1-R25 (2011).

\section{Author Contributions}

The project was conceived and supervised by V.T. and V.G.P. B.L., M.H.P., and R.A.A. performed assembly of the RTD embedded CR2032 cells and other laboratory experiments. V.T., V.G.P., T.E.A. and C.T.L. provided insights on customized LIB optimization, experiment analysis and data interpretation. B.L. and M.H.P. prepared the main manuscript text. V.T., V.G.P., T.E.A. and C.T.L. reviewed and finalized the manuscript. All authors have contributed with edits, seen, and approved the manuscript.

Additional Information

Supplementary information accompanies this paper at https://doi.org/10.1038/s41598-019-49616-w.

Competing Interests: The authors declare no competing interests.

Publisher's note: Springer Nature remains neutral with regard to jurisdictional claims in published maps and institutional affiliations. 
(c) (i) Open Access This article is licensed under a Creative Commons Attribution 4.0 International License, which permits use, sharing, adaptation, distribution and reproduction in any medium or format, as long as you give appropriate credit to the original author(s) and the source, provide a link to the Creative Commons license, and indicate if changes were made. The images or other third party material in this article are included in the article's Creative Commons license, unless indicated otherwise in a credit line to the material. If material is not included in the article's Creative Commons license and your intended use is not permitted by statutory regulation or exceeds the permitted use, you will need to obtain permission directly from the copyright holder. To view a copy of this license, visit http://creativecommons.org/licenses/by/4.0/.

(C) The Author(s) 2019 